\title{
Natural convection heat transfer from a thermal heat source located in a vertical plate fin ${ }^{2}$
}

\author{
Moghtada Mobedi ${ }^{\mathrm{a}}$, Bengt Sunden ${ }^{\mathrm{b}, *}$ \\ ${ }^{a}$ Mechanical Engineering Department, Izmir Institute of Technology, Urla 35430, Izmir, Turkey \\ ${ }^{\mathrm{b}}$ Division of Heat Transfer, Lund Institute of Technology, Lund, Sweden
}

Available online 17 May 2006

\begin{abstract}
A steady state conjugate conduction-convection investigation is performed on vertical plate fin in which a small heat source is located. Heat from the fin surface is transferred to the surroundings by laminar natural convection. The governing equations for the problem are the heat conduction equation for the fin and the boundary layer equations, which are continuity, momentum and energy equations, for the fluid. A computer program is written by using the finite difference method in order to solve the governing equations which are nonlinear and coupled. The best location of the heat source in the fin for maximum heat transfer rate depends on two parameters which are the conduction-convection parameter and the Prandtl number. The obtained results have shown that for the fin with large conduction-convection parameter, a heat source location for maximum heat transfer rate exists.
\end{abstract}

(C) 2006 Elsevier Ltd. All rights reserved.

Keywords: Natural convection; Conjugate conduction-convection; Vertical fins

\section{Introduction}

Vertical plate fins are widely employed in natural convection in order to increase heat transfer rate. Simple theoretical analysis related to this type of fin can be found in heat transfer textbooks but the temperature distribution along the fin is obtained based on the average heat transfer coefficient. However, accurate results for the fin temperature and heat transfer rate should be obtained by considering variation of the local heat transfer coefficient along the fin and variation of the fin temperature in determination of the fluid temperature. The conjugate solution of the heat conduction equation for the fin and continuity, momentum and energy equations for the flow provides an accurate solution for the natural convection fin problem.

Among theoretical studies for a vertical plate fin, Sparrow and Acharya [1] were probably the first ones who performed a conjugate conduction-convection analysis. They studied the interaction between local convective heat transfer and heat conduction in the fin. In their problem, the upper end of the fin is attached to a heat source whose temperature is different from the ambient temperature. They compared the numerical results of the conjugate solutions with the results of

\footnotetext{
放 Communicated by W.J. Minkowycz.

* Corresponding author.

E-mail address: bengt.sunden@vok.lth.se (B. Sunden).
} 


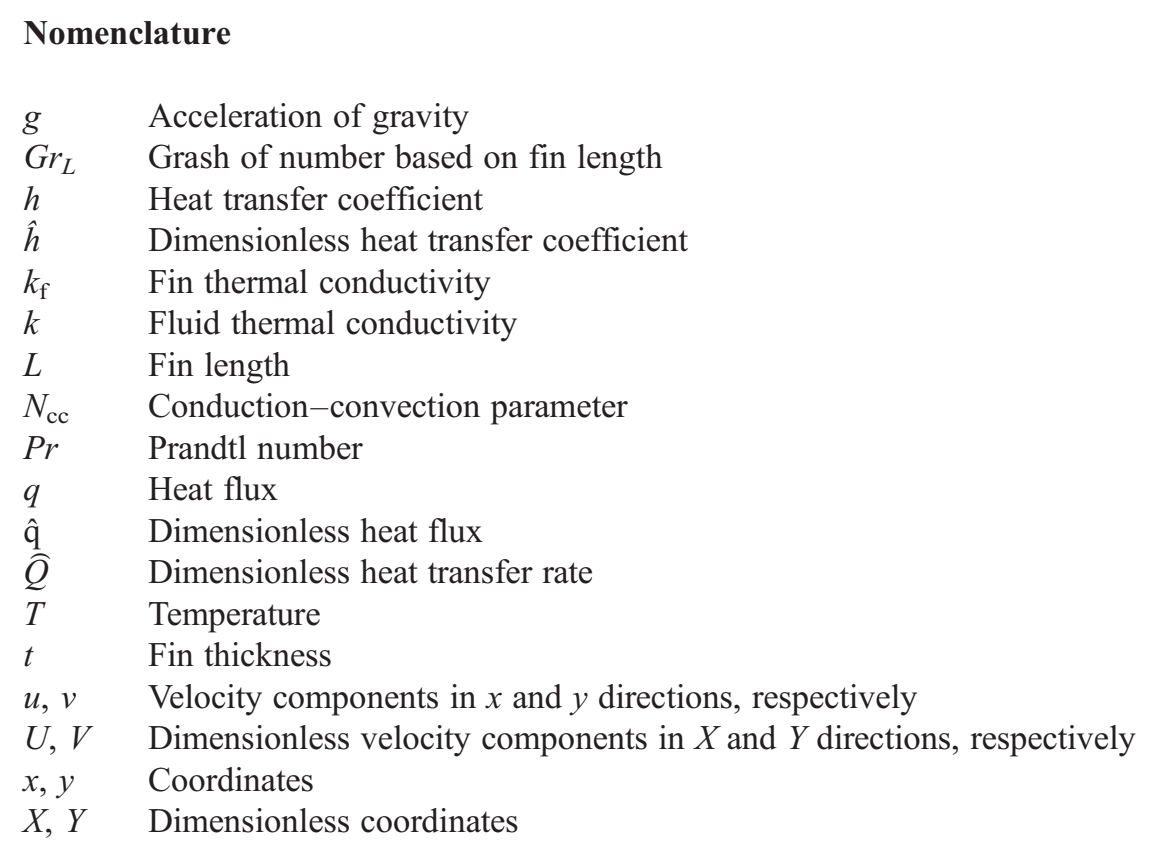

\section{Greek Symbols}

$\beta \quad$ Thermal expansion coefficient

$v \quad$ Kinematic viscosity

$\theta \quad$ Dimensionless temperature

$\xi \quad$ Dimensionless heat source location

\begin{tabular}{ll}
\multicolumn{2}{l}{ Subscripts } \\
o & Heat source \\
$\mathrm{f}$ & Fin \\
$\infty$ & Ambient
\end{tabular}

conventional fin theories. Sunden has extended the studies of Sparrow and Acharya, see Refs. [2,3]. He performed two studies for this type of fin for laminar and/or turbulent convection and mixed convection heat transfer. Pop and Hossain [4] have carried out a conjugate study of natural convection flow along a vertical plate fin embedded in a porous medium saturated either pure or saline water. In their study, an implicit finite difference scheme with the Keller box method was employed to solve the boundary layer equations which were written in terms of stream function. They investigated the effect of the conduction-convection parameters on temperature field, heat transfer coefficient and heat flux from the fin. Mobedi et al. [5] have solved a conjugate conduction-natural convection heat transfer problem for a rectangular fin attached to a partially heated horizontal base. They carried out their study for air when the flow is laminar and steady. Recently, Trevino et al. [6] have performed a conjugate conduction-free convection study on a rectangular vertical fin which is heated from above. They studied the thermal penetration length over which the temperature of a fin decreases from its maximum value at the top of the fin to the ambient temperature.

\section{Problem under consideration}

In this study, the analyzed fin is a vertical plate fin with thickness $t$ and length $L$ as illustrated in Fig. 1. A heat source, whose temperature $T_{\mathrm{o}}$, exceeds the ambient temperature $T_{\infty}$, is placed in the fin. The diffused heat 


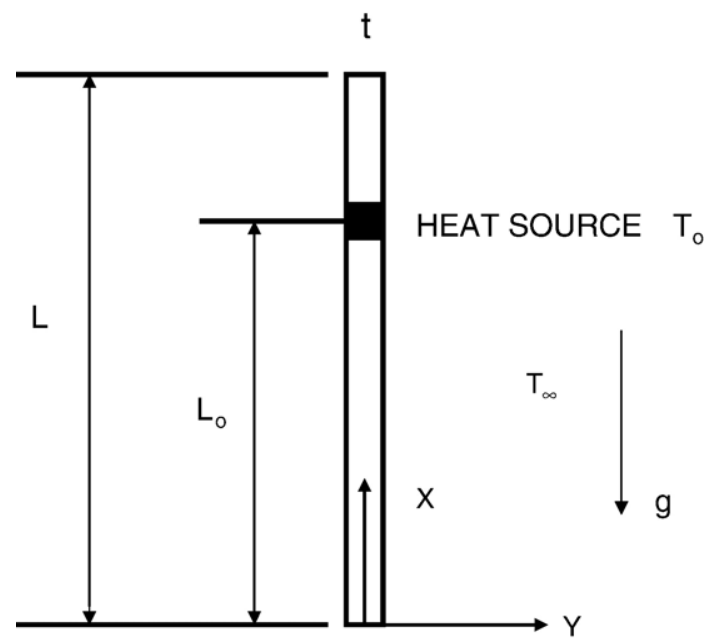

Fig. 1. A schematic view of the considered vertical plate fin with a heat source.

throughout the fin is transferred to the surroundings only by laminar natural convection. The thickness of the fin is small, thus, the heat conduction throughout the fin is considered one-dimensional. The height of the heat source is assumed negligible; however its thickness is the same as the fin thickness. The heat transfer from the tip and end of the fin is omitted. The study is performed for air with $\operatorname{Pr}=0.7$.

\section{Governing equations}

With the Boussinsq approximation, the dimensionless form of the conservation equations for the flow can be written as:

$$
\begin{aligned}
& \frac{\partial U}{\partial X}+\frac{\partial V}{\partial Y}=0 \\
& U \frac{\partial U}{\partial X}+V \frac{\partial U}{\partial Y}=\frac{\partial^{2} U}{\partial Y^{2}}+\theta \\
& U \frac{\partial \theta}{\partial X}+V \frac{\partial \theta}{\partial Y}=\frac{1}{P r} \frac{\partial^{2} \theta}{\partial Y^{2}}
\end{aligned}
$$

The above equations are nondimensionlized by employing the following dimensionless parameters:

$$
\begin{aligned}
& X=x / L ; Y=(y / L) G r_{L}^{1 / 4} ; U=u /\left(g \beta\left(T_{0}-T_{\infty}\right) L\right)^{1 / 2} ; V=v /\left(g \beta\left(T_{0}-T_{\infty}\right) v^{2} / L\right)^{1 / 4} ; \\
& \theta=\left(T-T_{\infty}\right) /\left(T_{0}-T_{\infty}\right) \quad G r_{L}=g \beta\left(T_{0}-T_{\infty}\right) L^{3} / v^{2}
\end{aligned}
$$

The dimensionless form of the heat conduction equation for the fin can be obtained as:

$$
\frac{\mathrm{d}^{2} \theta_{\mathrm{f}}}{\mathrm{d} X^{2}}=N_{\mathrm{cc}} \hat{h} \theta_{\mathrm{f}}
$$

where $\theta_{\mathrm{f}}, N_{\mathrm{cc}}$ and $\hat{\mathrm{h}}$ are dimensionless fin temperature, dimensionless conduction-convection parameter and dimensionless local convection heat transfer coefficient, respectively and they are defined as:

$$
\theta_{\mathrm{f}}=\left(T_{\mathrm{f}}-T_{\infty}\right) /\left(T_{0}-T_{\infty}\right) .
$$




$$
\begin{aligned}
& N_{\mathrm{cc}}=\frac{k L}{k_{\mathrm{f}} t} G r_{L}^{1 / 4} \\
& \hat{h}=\frac{h L}{k G r_{L}^{1 / 4}}=\left(-\frac{\partial \theta}{\partial Y} / \theta\right)_{Y=0}
\end{aligned}
$$

In the present investigation, the following boundary conditions are used for the boundary layer equations:

$$
\begin{array}{ll}
Y=0 & U=V=0, \theta=\theta_{\mathrm{f}}(X) \\
Y \rightarrow \infty & U=0, \theta=0 \\
X=0 & U=0, \theta=0
\end{array}
$$

The boundary conditions for the fin heat conduction equation, Eq. (6), are:

$$
\begin{aligned}
& X=0 \quad \frac{\mathrm{d} \theta_{\mathrm{f}}}{\mathrm{d} X}=0 \\
& \xi=L_{0} / L \quad \theta_{\mathrm{f}}=1 \\
& X=1 \quad \frac{\mathrm{d} \theta_{\mathrm{f}}}{\mathrm{d} X}=0
\end{aligned}
$$

As it can be seen from the dimensionless form of the governing equations and boundary conditions, the results of the problem depend on three dimensionless parameters, namely $\operatorname{Pr}, N_{\mathrm{cc}}$ and $\xi$. The variation of $\operatorname{Pr}$ number is not considered since the study is performed only for air with Prandtl number $P r=0.7$. Therefore, for the present study the number of effective parameters is reduced to two which are $N_{\mathrm{cc}}$ and $\xi$. Based on dimensionless variables which are presented in Eq. (4), the dimensionless local heat flux along the fin can be determined by the following relation:

$$
\hat{q}=\frac{q L}{k\left(T_{0}-T_{\infty}\right) G r_{L}^{1 / 4}}=-\left.\frac{\partial \theta}{\partial Y}\right|_{Y=0}
$$

The dimensionless total heat transfer rate from both sides of the fin can be calculated by integrating the local heat flux along the fin:

$$
\hat{Q}=2 \int_{X=0}^{X=1}\left(-\left.\frac{\partial \theta}{\partial Y}\right|_{Y=0}\right) \mathrm{d} x
$$

\section{Numerical solution procedure}

The continuity, momentum and energy equations are solved by using the finite difference method which is explained in Ref. [7]. The nonlinearities of the momentum and energy equations are removed by adopting $U$ and $V$ values of the previous iteration step. A first order upwind scheme is employed to obtain the finite difference form of the convective terms in the momentum and energy equations. The set of numerical equations for momentum and energy equations is solved by the Thomas Algorithm method line by line in $Y$ direction and marching in the $X$ direction. A successive under-relaxation value is adapted to control the speed of propagation of $U$ and $\theta$ in the computational domain. The value of SUR (successive under relaxation) is kept around 0.10.3. The heat conduction equation of the fin is solved by finite differences using Gauss-Seidel iteration method.

The convergence of the solution procedure involves the convergence of inner iteration (for the solution of boundary layer equations and fin conduction equation) and outer iteration which is the convergence of the whole 
procedure. For the convergence of the boundary layer equations, the following convergence criterion is employed;

$$
\left|\frac{\hat{h}^{n+1}-\hat{h}^{n}}{\hat{h}_{\max }^{n}}\right| \leq 10^{-10}
$$

while the convergence criterion for the heat conduction equation is set as;

$$
\left|\frac{\theta_{\mathrm{f}}^{n+1}-\theta_{\mathrm{f}}^{n}}{\theta_{\mathrm{f}_{\max }}^{n}}\right|_{\text {max }} \leq 10^{-10}
$$

For the outer iteration, the solution of the whole procedure is said to be converged when the relative rate of changes of normalized dimensionless temperature at each node inside the fin does not exceed $10^{-7}$, i.e.

$$
\left|\frac{\theta_{\mathrm{f}}^{n+1}-\theta_{\mathrm{f}}^{n}}{\theta_{\mathrm{fmax}}^{n}}\right|_{\max } \leq 10^{-7}
$$

The numerical tests for the location of the open boundary $(Y \rightarrow \infty)$ have shown that, $7 L$ is a sufficient distance for the location of the open boundary from the fin. A non-uniform mesh is used for the fin and boundary layer domains. The grid sizes are taken fine around the fin and expanded continuously towards the far boundary. The grid sizes also expanded from the leading edge to the top of fin. The size of the grids at the leading edge has an important effect on the accuracy of the results. Due to the high temperature gradient around the heat source, smaller grid sizes are also used around the heat source. Numerical tests have shown that 500 nodes along fin and 300 nodes in the $Y$ direction are sufficient to obtain the accurate computational results. For all runs which are performed in this investigation, the minimum and maximum grid sizes in the boundary layer domain and fin are $\Delta X_{\min }=1.08 \times 10^{-8}$ and $\Delta X_{\max }=2 \times 10^{-2}$ for the $X$ direction and $\Delta Y_{\min }=10^{-3}$ and $\Delta Y_{\max }=0.15$ for the $Y$ direction. A finite difference program which is written in Fortran language is used to solve the set of the governing equations. In order to evaluate the dimensionless local heat transfer coefficient and heat flux along the fin, a second order finite difference approximation is used to calculate the temperature gradient for the fluid adjacent to the fin.

\section{Results}

For establishing the accuracy of the employed solution method and computer program, calculations for the isothermal vertical plate $\left(N_{\mathrm{cc}}=0\right)$ were carried out and the results were compared with the results of the similarity solution reported in the literature. The value of dimensionless parameter $N u_{\mathrm{x}} / G r_{\mathrm{x}}^{1 / 4}$ for the different nodes along the fin is obtained as 0.357 which is the same as the value reported in Ref. [8]. The problem which has been handled by Sparrow and Acharya [1] was also solved. A good agreement between the results obtained by the present study and the results reported by Sparrow and Acharya [1] was observed.

Based on obtained results for temperature distribution along the fin for different heat source locations, it is observed that for the fins with the value of $N_{\mathrm{cc}}$ close to zero, the fin temperature is almost constant and the fin can be considered as an isothermal plate as can be seen from Fig. 2 in which the variation of dimensionless fin temperature for $\xi=0.75$ heat source location and different $N_{\mathrm{cc}}$ value are shown. Mathematically, the decrease of $N_{\mathrm{cc}}$ value reduces the influence of the right-hand side of Eq. (5) in the solution. Because the adiabatic boundary conditions are applied to the tip and end of the fin, the solution of Eq. (5) approaches a constant value which is the temperature of the heat source. The expected result for the fin with large $N_{\mathrm{cc}}$ value is a considerable temperature variation along the fin. For fins with large $N_{\mathrm{cc}}$ values, the fin temperature monotonically decreases from the heat source location towards the tip and end of the fin. At the heat source location, the maximum fin temperature prevails (Fig. 2).

The distribution of the dimensionless local heat transfer coefficient along the same fin of Fig. 2 is shown in Fig. 3. For small $N_{\mathrm{cc}}$ values, the distribution of $\hat{\mathrm{h}}$ is very similar to the distribution of the local heat transfer coefficient for an isothermal plate, because the fin temperature is almost constant. It is observed that for fins with small $N_{\mathrm{cc}}$ values, changes in the location 


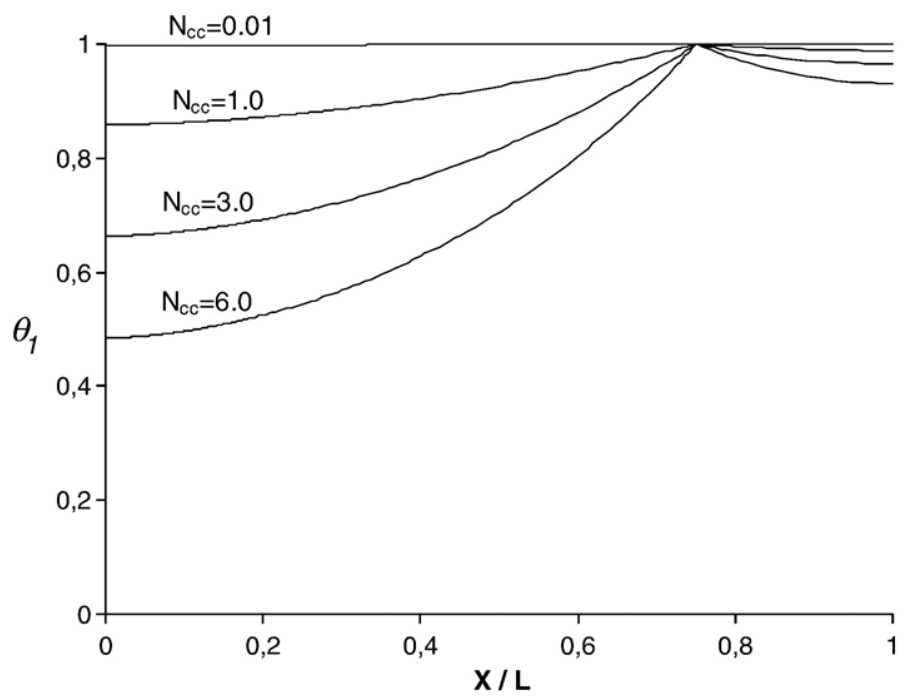

Fig. 2. The dimensionless temperature distribution along the fin with the heat source location at $\xi=0.75$ for four different $N_{\mathrm{cc}}$ values.

of the heat source do not affect the local heat transfer coefficient. For large $N_{\mathrm{cc}}$ values, the distribution of the local heat transfer coefficient becomes different. It deceases monotonically from the tip of the fin, reaches a minimum and then starts to increase to its value at the heat source location. Beyond the maximum at the heat source location, it monotonically decreases towards the fin end. This variation of the local heat transfer coefficient can be explained by considering fin temperature distribution. For large $N_{\mathrm{cc}}$ values, the non-uniform wall temperature distribution causes enhancement of the buoyancy force and increase of the local heat transfer coefficient at the heat source location.

The variation of heat flux along the fin is very similar to the local heat transfer coefficient variation, because for fins with small $N_{\mathrm{cc}}$, the fin temperature is constant, the heat flux distribution does not vary by changing the location of the heat source. For fins with large $N_{\mathrm{cc}}$ values, due to enhancement of the buoyancy force at the heat source location, the distribution of the heat flux is affected by changing the location of the heat source. By increasing the $N_{\mathrm{cc}}$ value and particularly for the cases that the heat source is located after the middle of the fin, a sharp drop of the heat flux at the region near the leading edge of the fin attracts

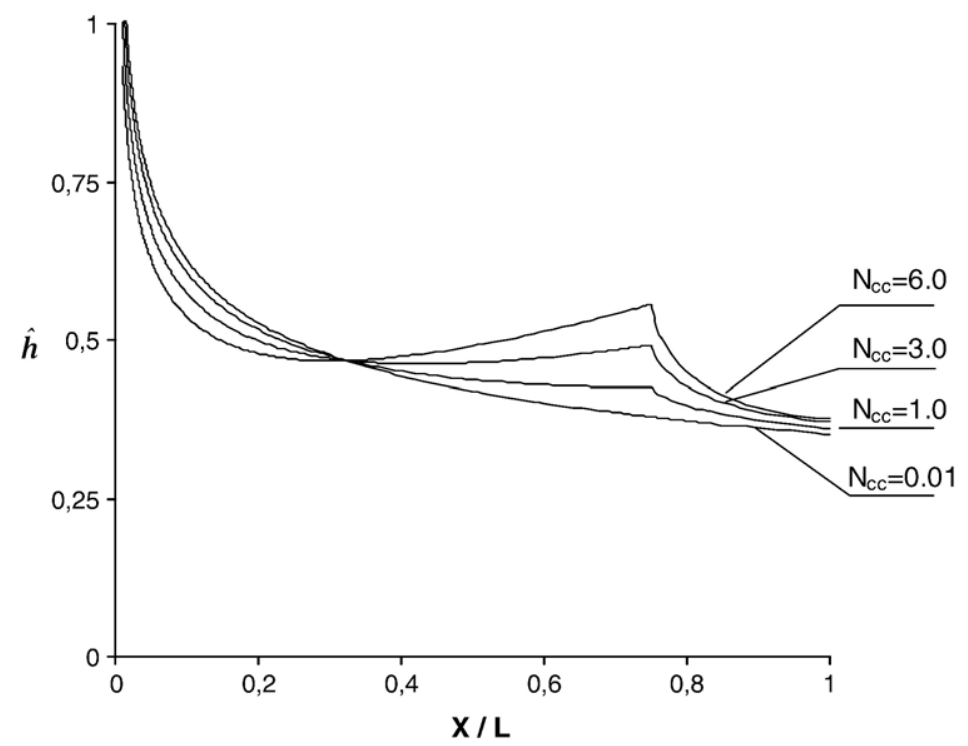

Fig. 3. The distributions of dimensionless local heat transfer coefficient along the fin with heat source location at $\xi=0.75$ for four different $N_{\mathrm{cc}}$ values. 


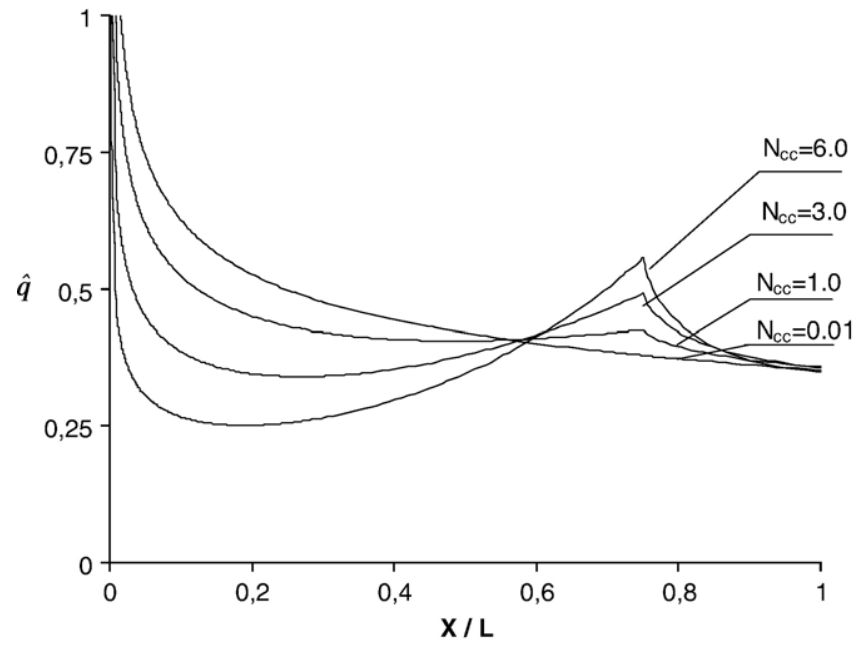

Fig. 4. The distribution of dimensionless heat flux along the fin with heat source location at $\xi=0.75$ for four different $N_{\mathrm{cc}}$ values.

attention as it is seen from Fig. 4. Both the decrease of the wall temperature from the heat source location towards the fin tip and the decrease of the local heat transfer coefficient at the fin tip region cause the heat flux at the region near the leading edge to drop steeply.

Fig. 5 shows the variation of the total heat transfer rate from both sides of the fin with the location of the heat source for different $N_{\text {cc }}$ values. For a small conduction-convection parameter, the heat transfer rate is not influenced by changing the location of the heat source. This is an expected result for fins with small $N_{\mathrm{cc}}$ because the fin temperature is very uniform. The maximum heat transfer rate and consequently the maximum fin performance always occur for the fins with small $N_{\mathrm{cc}}$ values, because the fin temperature is almost equal to that of the heat source. For large $N_{\mathrm{cc}}$ values, by changing the location of the heat source from the tip toward the end of the fin, a heat source location for maximum heat transfer rate appears. The distribution of the heat flux through the fin causes this behavior of the heat transfer rate versus the heat source location. When the heat source is located at the leading edge, the heat flux decreases monotonically towards the fin end. By changing the place of the heat source and moving it to the middle of the fin, increases of the heat flux from the tip of the fin towards the heat source location occur due to the increase of the temperature. This can be seen from Fig. 6 where the variations of the heat flux along a fin with $N_{\mathrm{cc}}=6$ for different heat source locations are shown. However, after middle of the fin as the heat source comes close to the fin end, the downward slope of the heat flux at the leading edge region becomes steeper. This has a

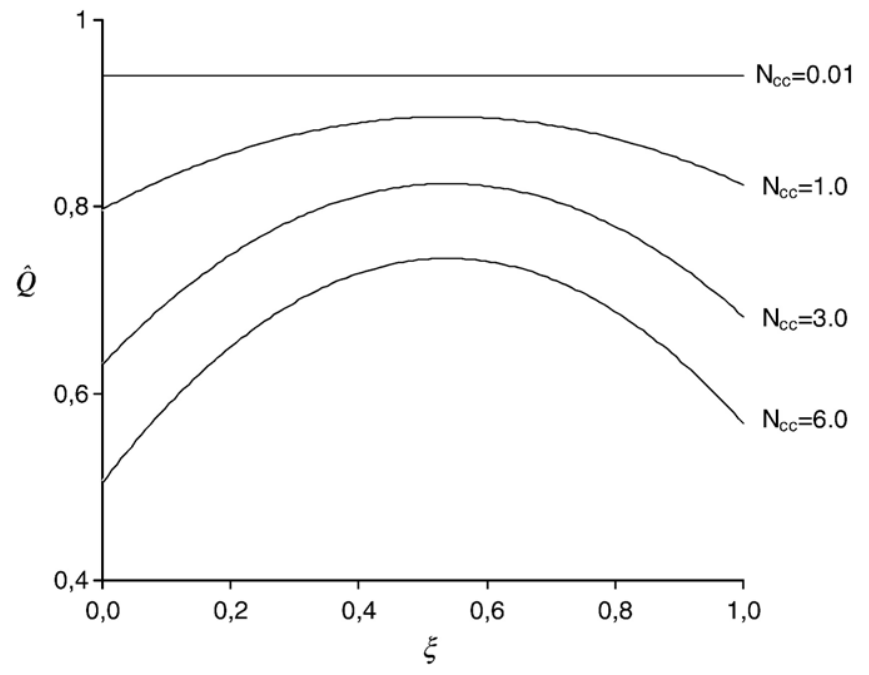

Fig. 5. Variation of dimensionless heat transfer rate with location of the heat source for different conduction-convection parameter. 


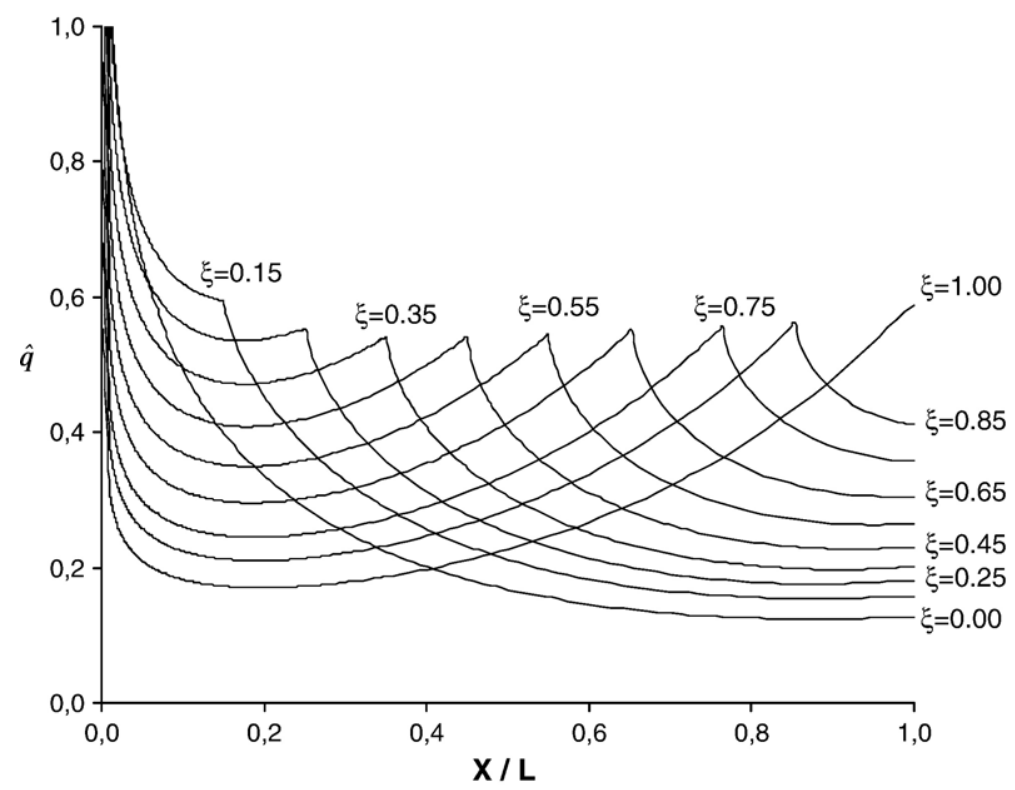

Fig. 6. Variation of the dimensionless heat flux with heat source location for a fin with $N_{\mathrm{cc}}=6$.

negative effect on the increasing trend of heat transfer rate and consequently the heat transfer rate decreases at the region near the fin end. Based on various numerical results, which are obtained for different heat source locations and different $N_{\mathrm{cc}}$ values, the maximum heat transfer rate and fin performance are obtained when the heat source is approximately located at $\xi=0.54$.

\section{Conclusion}

A conjugate conduction-convection study was performed on a vertical plate fin in which a small heat source was located. For a small conduction-convection parameter, the fin behaves like an isothermal vertical plate and the heat transfer rate from the fin does not vary by changing the location of the heat source. However, for fins with large values of the conduction-convection parameter, the heat transfer rate is influenced by the location of heat source. By changing the location of the heat source from tip towards the end of the fin, a location for maximum heat transfer rate was found. The obtained numerical results showed that for fins with large conduction-convection parameter $\xi=0.54$ is approximately the best location for the heat source because it provides maximum heat transfer from the fin.

\section{References}

[1] E.M. Sparrow, S. Acharya, A natural convection fin with a solution-determined nonmonotonically varying heat transfer coefficient, ASME, Journal of Heat Transfer 103 (1981) 218-225.

[2] B. Sunden, Conjugate mixed convection heat transfer from a vertical rectangular fin, International Communications in Heat and Mass Transfer 10 (4) (1983) 267-276.

[3] B. Sunden, Analysis of conjugated laminar and turbulent forced convection-conduction heat transfer of a plate fin, International Communications in Heat and Mass Transfer 16 (6) (1989) 821-831.

[4] I. Pop, M.A. Hossain, Conjugate natural convection from a vertical plate fin in a porous medium saturated with cold water, in: B. Sunden, E. Blums, A. Zukauskas (Eds.), Advances in Engineering Heat Transfer, Computational Mechanics Publication, Southampton, 1995, pp. 161-170.

[5] M. Mobedi, A. Saidi, B. Sunden, Computation of conjugate natural convection heat transfer from a rectangular fin on a partially heated horizontal base, Heat and Mass Transfer 33 (1998) 333-336.

[6] C. Trevino, E. Luna, F. Mendez, F.J. Higuera, Natural convective conjugate cooling mechanism in vertical fins, AIAA Journal of Thermophysics and Heat Transfer 17 (3) (2003) 396-401.

[7] Y. Jaluria, K. Torrence, Computational Heat Transfer, Second ed.Taylor and Francis, 2003.

[8] W.M. Kays, M.E. Crawford, Convective Heat and Mass Transfer, Third ed., McGraw-Hill, 1993, p. 402. 